\title{
The prevalence of orthodontic extractions
}

\author{
Jane A. Weintraub, DDS, MPH, ${ }^{a}$ Peter S. Vig, BDS, PhD, D. Orth., FDSRCS (Eng.), ${ }^{D}$ \\ Colleen Brown, DDS, MS, ${ }^{\mathrm{c}}$ and Charles J. Kowalski, PhD ${ }^{\mathrm{d}}$ \\ Chapel Hill, N.C., Ann Arbor, Mich., and Berkeley, Calif.
}

\begin{abstract}
The controversy regarding extractions for othodontic treatment continues and today is invoked by some as a feature of the "standard of care." For this and other reasons, it is necessary to have contemporary data on the general prevalence of orthodontic extractions and the interpractice range of extraction rates in current specialty practice and, if possible, also to establish possible reasons for, and consequences of, the extraction versus nonextraction decision. Although all treatment decisions are made on a case-by-case basis, practitioners appear to have personal preferences for or against extractions and hence adopt policies with different degrees of aversion to or bias toward extraction therapy. A number of factors enter into the extraction decision. These include features of the malocclusion, objectives of treatment, and the technique selected to accomplish desired results. The present study does not address such issues but merely serves to provide epidemiologic data to estimate extraction frequency in contemporary orthodontic practice. Accordingly, a telephone survey of all licensed orthodontists in Michigan was conducted to determine their subjective estimates of extraction rates for patients in their practices. There were 238 respondents, for a response rate of $90.2 \%$. Reported rates range from $5 \%$ to $87 \%$. We then selected five practices from the extremes of the reported extraction rates. Three practices from the high end and two from the low end were included for an examination of patient records. Records of a total of 438 patient whose treatment had been completed were reviewed. The actual extraction rates for these practices ranged from $25 \%$ to $85 \%$, which differed considerably from the clinicians' subjective estimates. Differences ranged from an overestimate of about $20 \%$ to a $15 \%$ underestimate. Results from the survey indicate that a wide range of extraction frequency exists among orthodontists. The pilot study of the actual extraction rates suggests that discrepancies may exist between perceived and actual extraction rates. To obtain reliable estimates of extraction rates, it is therefore suggested that random sampling of a larger number of practices and objective evaluation of patient records are required rather than the use of questionnaires. (AM J ORTHOD Dentofac ORTHOP 1989;96:462-6.)
\end{abstract}

$\mathrm{T}$ orthodontic treatment has been a topic of discussion and a source of clinical disagreement for many years. Each clinician can obviously justify extraction or nonextraction decisions, case by case, particularly on a post hoc basis and specifically by demonstrating good treatment results with either option. Despite or possibly because of this, practitioners appear to have personal preferences either for or against extractions and hence adopt policies with different degrees of aversion to or affinity for extraction therapy. On an individual basis, a number of factors enter into the extraction decision. These include, among others, etiologic and morpho-

\footnotetext{
${ }^{a}$ Assistant Professor, Department of Dental Ecology, School of Dentistry, University of North Carolina, Chapel Hill.

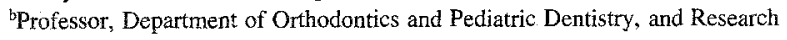
Scientist, Center for Human Growth and Development, School of Dentistry, The University of Michigan, Amn Arbor.

In private orthodontic practice, Berkeley, Calif.

${ }^{d}$ Professor, Department of Biological and Materials Science, and Research Scientist, Dental Research Institute, The University of Michigan, Ann Arbor. $18 / 1 / 10454$
}

logic features of the malocclusion, specific objectives of the treatment, and the techniquc selected to accomplish the desired result. The relationships between treatment strategies and outcomes has not yet been subjected to rigorous analysis according to objective criteria employed in clinical epidemiology, and controlled clinical trials have yet to establish the relative efficacy of extraction or nonextraction strategies, for either short- or long-term results of orthodontic treatment. It is fair to say that, to date, orthodontists and other dentists who treat malocclusions base their extraction decisions on personal experience and preference.

In spite of the empirical basis for the clinical policies of proponents and opponents of orthodontic extractions, claims for the inherent superiority of such opposing policies are made, and at present this controversy has even become a part of the current interest focused on the "standard of care" that is appropriate for providers of orthodontic care. Whether a particular frequency of extractions conforms to the standard, defined as being within the usual range or not, can be established only 
Table I. Summary of reported frequency of extraction of permanent teeth for orthodontic treatment purposes

\begin{tabular}{c|c|l|l}
\hline Extraction treatment (\%) & Sample size & \multicolumn{1}{|c|}{ Sample description } & \multicolumn{1}{c}{ Publication } \\
\hline 6.5 & - & Private patients, U.S.A. & Case (1913) \\
8.0 & 200 & Private patients, Ireland & Friel (1931) \\
15.9 & 1000 & Clinic patients, German & Breeg (1963) \\
17.4 & 7188 & Clinic patients, Poland & Majchrowicz (1972) \\
19.2 & 1019 & Clinic patients, Czechoslovakia & Adam (1968) \\
23.1 & 1144 & Clinic patients, Czechoslovakia & Andrik et al. (1975) \\
23.2 & 203 & Clinic patients, Poland & Dyras (1973) \\
27.8 & 1000 & Clinic patients, Germany & Bredy and Reichel (1971) \\
733.3 & - & Private patients, U.S.A. & Ricketts (1976) \\
75.0 & 1000 & Private patients, England & Rose (1973) \\
80.0 & - & Private patients, U.S.A. & Tweed (1966) \\
42.5 & 1189 & Clinic patients, England & Hooper (1967)
\end{tabular}

From Pcck S, Peck H. AM J ORTHOD 1979;76:491-6.

if data exist on the pattern of contemporary orthodontic practice.

The lack of clinical agreement and the wide range of extraction treatment frequency has been reported previously by Perlow, ${ }^{1}$ Salzmann, ${ }^{2}$ and Peck and Peck, ${ }^{3}$ whose summary of the reported extraction frequencies from 13 sources in the literature is reprinted in Table I and reveals a frequency range from $6.5 \%$ to $83.5 \%$. Their data span a period that ranges between 1913 and 1979 and thus may not be representative of contemporary practice.

The purpose of our study was to quantify current orthodontic practice trends with respect to the extraction decision. This preliminary investigation was not designed to evaluate the basis for or the merits of the decision. Although information on the efficacy of orthodontic treatment alternatives is clearly desirable, such future studies require preliminary work to provide relevant epidemiologic characteristics of the salient features and the major trends in clinical practice. Hence, studies such as reported here are not only required to provide prevalence estimates of practice trends but are also useful to enable the refinement of methods and the formulation of specific hypotheses for subsequent work dealing with clinical efficacy.

\section{MATERIALS AND METHODS}

The initial phase of this two-part study consisted of a telephone survey of all the orthodontists in Michigan. The Orthodontic Directory of the World ${ }^{4}$ was used to identify and locate this population. Attempts were made to contact all 264 Michigan orthodontists by telephone during late 1986 and early 1987 . The survey consisted of one question, which was asked by one interviewer: "What is the estimate of the percentage of extraction versus nonextraction treatment in your practice?"
If an answer was given with a range (for example, $15 \%$ to $20 \%$ ), this was recorded as such, but was later analyzed as an average of the two figures (e.g., $15 \%$ to $20 \%=17.5 \%$ ). The orthodontists' professional schools and their year of graduation, as published in the Directory, and any additional comments that they made were recorded.

After completion of the telephone survey, the practices at the two extremes of estimated extraction rate were identified. Five practices were selected from these two extremes for detailed record reviews of debanded patients to compare the actual and estimated extraction rates. The primary criteria for othodontic practice selection were a high or low self-reported extraction rate, proximity of a practice to Ann Arbor, and willingness of the practitioner to participate in the study.

In four of the practices, recently debanded patients were selected for the record review. The patients in practice 5 , however, were treated over a wide range of years, with debandings from 1947 to 1984 . This practice was selected because of the complete records available in addition to the fact that it satisfied the other criteria for selection.

\section{Statistical considerations}

Frequency distributions for the telephone survey responses were calculated for extraction versus nonextraction rates. Possible associations between these responses and the year of graduation, the number of years in practice, and the school granting the orthodontic degree or certificate were investigated with the use of correlation coefficients. The main concern of this study was the data from the telephone survey. Practices were visited primarily to check on the accuracy of the selfreported data. Practices from the extremes of the range of reported extraction rates were selected because we 


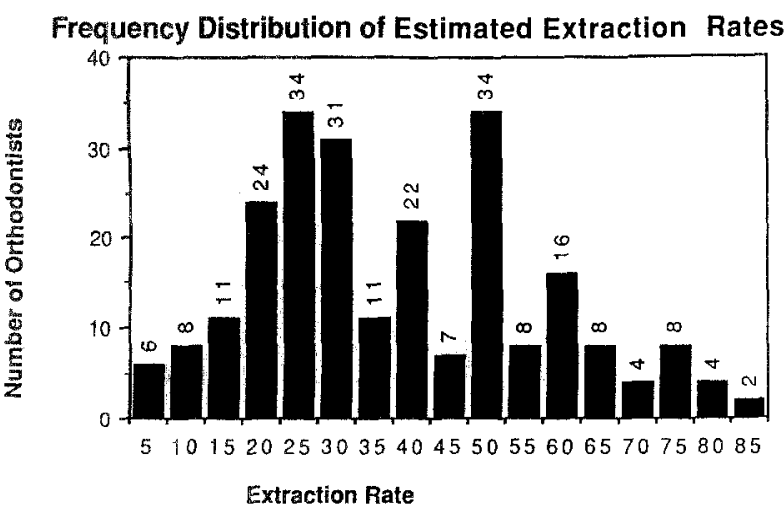

Fig. 1. Frequency distribution of self-reported exlraclion rates by Michigan orthodontists in 1987.

were interested in whether these could be considered as "outliers" or represented "real data."

\section{RESULTS}

\section{Survey of orthodontists}

Answers were obtained from 238 of the 264 Michigan orthodontists, representing a $90 \%$ response rate. Thesc orthodontists were graduates of 32 different university programs. Just over half $(122$, or $51.3 \%)$ attended the University of Michigan, and the next largest group (36, or $15.1 \%$ ) attended the University of Detroit. Sixteen of the 32 schools were represented by just one orthodontist. Almost all of the 32 schools were located on the East Coast or in the Midwest. The year of graduation from an orthodontic program ranged from 1939 to 1985 , although this distribution was skewed toward more recent years. The median year of graduation was 1971.

The distribution of estimated extraction rates is presented in Fig. 1. The average of estimates for the rate of extraction was $39 \%$ for the entire group of orthodontists, with a range of $5 \%$ to $87.5 \%$ (reported as " $85 \%$ to $90 \%$ ") and a standard deviation of $18.3 \%$.

There was no correlation between extraction rate and year of graduation from an orthodontic program either for the entire group $(r=-0.09)$ or for the 122 University of Michigan graduates $(r=0.07)$. There were 18 respondents who reported an estimated rate of $70 \%$ or higher and 25 who reported an estimated rate of $20 \%$ or less. The five orthodontists selected for the second part of the study had estimated their extraction rates to be $10 \%, 15 \%$ to $20 \%, 70 \%$ to $80 \%, 75 \%$, and $85 \%$ to $90 \%$. This group, therefore, had two representatives from the low end of the range of estinnated extraction rates and three from the high end.
Table Il. Extraction rates: Estimates compared to actual rates for five practices in southeastern Michigan

\begin{tabular}{c|c|c|c}
\hline & $\begin{array}{c}\text { Estimated rate } \\
\text { (telephone survey) } \\
(\%)\end{array}$ & $\begin{array}{c}\text { Difference between } \\
\text { actual and } \\
\text { estimated rates } \\
\text { Actual rate } \\
(\text { rectual restimate }) \\
(\%)\end{array}$ \\
\hline 1 & 10 & 25.0 & +15.0 \\
2 & $70-80$ & 55.4 & -19.6 \\
3 & $85-90$ & 68.6 & -18.9 \\
4 & $15-20$ & 32.6 & +15.1 \\
5 & 75 & 85.4 & +10.4 \\
\hline
\end{tabular}

\section{Patient record review in five selected practices}

Records were reviewed by one of us for 438 debanded patients from the five practices in southeastern Michigan.

For these five practices, which were specifically selected because of their extreme rates, the actual mean rate of extractions was $54 \%$, which is higher than the $39 \%$ self-reported estimated rates for all Michigan orthodontists surveyed. These actual rates are compared to the self-reported estimated rates in the telephone survey in Table II. These five individual respondents' differences ranged from about a $20 \%$ overestimate to a $15 \%$ underestimate.

Four treatment patterns - either nonextraction or incisor, premolar, or second molar extraction-were found in the five practices and are summarized in Table III. Differences between the practices are evident. In practice 5 , only $13(6.5 \%)$ of the 201 total patients in the sample were treated without extractions. In contrast, practices 1 and 4 had only $5.2 \%(11 / 213)$ and $11.5 \%$ $(24 / 213)$, respectively, of the patients treated with premolar extractions, while they had $53.8 \%(7 / 13)$ and $30.8 \%(4 / 13)$ of the patients treated with second molar extractions.

\section{DISCUSSION}

The results of this survey show that there is little uniformity within this group of 238 orthodontists on the use of extractions in orthodontic treatment. Estimated extraction rates in their practices ranged from $5 \%$ to $87.5 \%$. The two variables considered in this survey-school and year of graduation from orthodontic training - showed no association with extraction rates. When a subset of graduates of one orthodontic program was evaluated, again there was no relationship 
Table III. Extraction patterns for five orthodontic practices in southeastern Michigan

\begin{tabular}{|c|c|c|c|c|c|c|c|c|c|}
\hline \multirow[b]{2}{*}{ Practice(s) } & \multirow[b]{2}{*}{$N$} & \multicolumn{2}{|c|}{ Nonextraction } & \multicolumn{2}{|c|}{ Incisor extractions } & \multicolumn{2}{|c|}{$\begin{array}{l}\text { Premolar } \\
\text { extractions }\end{array}$} & \multicolumn{2}{|c|}{$\begin{array}{l}\text { Second molar } \\
\text { extractions }\end{array}$} \\
\hline & & $\%$ & $\begin{array}{l}\text { No. of } \\
\text { patients }\end{array}$ & $\%$ & $\begin{array}{l}\text { No. of } \\
\text { patients }\end{array}$ & $\%$ & $\begin{array}{c}\text { No. of } \\
\text { patients }\end{array}$ & $\%$ & $\begin{array}{l}\text { No. of } \\
\text { patients }\end{array}$ \\
\hline $1-5$ & 438 & 46.1 & 201 & 2.1 & 9 & 48.9 & 213 & 3.0 & 13 \\
\hline 1 & 80 & 75.0 & 60 & 2.5 & 2 & 13.8 & 11 & 8.8 & 7 \\
\hline 2 & 93 & 44.6 & 41 & 3.3 & 3 & 51.1 & 48 & 1.1 & 1 \\
\hline 3 & 87 & 31.4 & 27 & 3.5 & 3 & 64.0 & 56 & 1.2 & 1 \\
\hline 4 & 89 & 67.4 & 60 & 1.1 & 1 & 27.0 & 24 & 4.5 & 4 \\
\hline 5 & 89 & 14.6 & 14 & 0 & & 85.4 & 75 & 0 & \\
\hline
\end{tabular}

between year of graduation and extraction rate, and the range is almost as large for this subset as it was for the entire data set $(8 \%$ to $85 \%)$. Orthodontists, either during their formal orthodontic education or after graduation, may be receiving conflicting information about whether or not teeth should be extracted for orthodontic purposes. While these variables show no relationship with estimated extraction rates, other variables not included in this study might be associated. These factors may include features of practice, such as the type of technique or techniques used, patient profile of the practice, and the orthodontist's attendance at continuing education courses.

This telephone survey represents responses from nearly all the orthodontists in Michigan, and it is not unreasonable to assume that the patient population of these practitioners is similar to orthodontic patients in practices throughout the United States. One may then conclude that the prevalence of orthodontic extractions among clinicians is extremely variable and is determined by factors other than the age of the clinician or the training received before graduation from a university-based specialty program.

This inevitably leads to the question of what factor or factors are responsible for the clinical decision to extract teeth in individual patients. Are such clinical decisions made in accordance with predetermined rationales? Are they internally consistent within individual practitioners? Are extractions mandated by the orthodontic technique of choice or by the conditions being treated? To what extent and how does the decision to extract or not to extract affect the dental position and facial appearance, which are generally considered to be attributes of treatment outcomes that clinicians assess for quality of result? Finally, are extraction rates significantly correlated with the posttreatment stability of treatment results among practices?
At present there are no data from rigorous clinical studies to answer any of these important questions. Conflicting views exist and, unfortunately, are based on subjective impressions and traditional orthodontic dogma derived from a variety of sources.

Since the 1944 "extraction panel" debate, ${ }^{5}$ it is safe to assume that the extraction decision has been greatly affected by changes and by a general proliferation in the treatment options available to orthodontists. Some of these changes, such as the use of functional appliances, have a direct impact on the issue of whether or not "basal" bone can be stimulated to grow. An example is provided by the Frankel appliance. ${ }^{6}$ This appliance is designed to keep the cheek muscles away from the developing dentition (with vestibular shields), thus allowing teeth to erupt vertically and "de-crowd." According to reports of clinical experience, notably that of Frankel himself and also of others, the use of this appliance leads to less extraction treatment. Watson, however, has pointed out that "there is insufficient data to prove its predictability," and whether the results so obtained differ in their stability from alternative approaches has not been rigorously tested.

Another orthodontic procedure closely related to the cxtraction/noncxtraction decision is palatal expansion. A number of different techniques are used to accomplish such expansion. One involves the use of a rapid palatal expander. Haas ${ }^{8}$ described this as a technique that is advantageous in the treatment of "selected arch length problems to avoid the profile disturbances so frequently associated with the removal of teeth." The use of this technique often eliminates the need for extractions to relieve crowding, as it expands the upper arch and thus creates more arch length, or space. Again, neither orthodontic nor orthopedic expansion techniques have been rigorously tested for efficacy in terms of success rate, predictability, or long-term stability. 
All five of the orthodontists involved in the present patient record survey estimated extraction rates that were quite different from the rates actually determined from the data. The average absolute difference between estimated rate and actual rate was $15.8 \%$. Part of the difference may be attributed to the fact that, in the telcphone survcy, orthodontists generally reported what they thought their current extraction rate was and the patients in the survey had all begun treatment earlier. Clinicians' inability to recall relative frequency of their choice from among treatment options can adversely affect subsequent, and dependent, clinical decisions. ${ }^{9}$ If the main guide for decision making is experience, then the reliability of clinicians' perceptions of their experience becomes important.

It is entirely unclear whether there is a current trend toward more or fewer extractions for orthodontic purposes occurring in the United States. It is known that among children tooth loss due to dental caries has been declining. ${ }^{10}$ The current study indicates that about half of the orthodontic patients have teeth extracted as part of their treatment. About $70 \%$ of this group had four premolars extracted. The rest had other combinations of teeth or fewer than four teeth extracted. For the 237 patients with extractions, there was a mean of 3.5 teeth extracted per person. This number is much higher than the mean number of missing teeth $(0.04)$ per 12-yearold child (which excludes orthodontic extractions) reported in the 1979-80 National Dental Caries Prevalence Survey. ${ }^{11}$ According to the same survey, ${ }^{12}$ more than 5 million children $(11.7 \%$ ) were currently or had been under orthodontic treatment. Although the goal of the dental profession in the United States today is to preserve teeth and prevent tooth loss, presumably teeth are extracted for orthodontic purposes for the long-term benefit of the entire dentition. The long-term results of extraction for orthodontic purposes, however, have not been studied in a controlled fashion. Even in the short term, there are some risks and potential side effects associated with surgical extraction in terms of time, costs, pain, and discomfort. The probabilities associated with the risks and benefits should be considered in the process of deciding whether or not to extract a tooth. Further investigation of how orthodontic treatment decisions are made and their long-term implications is needed. The principles of clinical epidemiology need to be applied to orthodontic research to augment the rationality of and provide a scientifically tenable basis for making extraction/nonextraction decisions.

\section{CONCLUSIONS}

1. The proportion of treatments in which they prescribed extractions (excluding third molars) was esti- mated by 238 Michigan orthodontists. Their individual estimates ranged from $5 \%$ to $87 \%$, with a mean of $39 \%$ $( \pm 18.3 \%)$

2. The frequency of self-reported extraction rates did not correlate with the orthodontists' age, the number of years in practice, or the university programs from which they graduated.

3. A review of the records of 438 patients from five selected practices suggests that clinicians' subjective estimates of the frequency with which they opt for extractions may differ from the actual rates.

4. Reliable data on the frequency distribution and prevalence of extractions, as well as other significant variables related to the pattern of orthodontics, are needed. Questionnaires asking for clinicians' subjective impressions of the frequency of treatment decisions may yield estimates that differ from objective counts. Results obtained from large-scale questionnaire surveys should be validated by objective estimates based on reviews of the records of patients from an appropriate subsample of practices.

\section{REFERENCES}

1. Perlow J. Extractions in orthodontics, updated. NY State Dent J 1976;42:415-9.

2. Salzmann JA. An evaluation of extraction in orthodontics. AM J ORTHOD 1965;51:928-9.

3. Peck $S$, Peck H. Frequency of tooth extraction in orthodontic treatment. AM J ORTHON 1979;76:491-6.

4. Oliver WH. Orthodontic directory of the world. Nashville: WH Oliver, 1986.

5. Hahn G. Extraction panel. Orthodontics: its objectives, past and present. AM J ORTHOD ORAL SURG 1944;20:401-4.

6. Frankel R. Decrowding during eruption under the screening influence of vestibular shields. AM J ORTHOD 1974;65:372-406.

7. Watson WG. An individual compass for extraction [Editorial]. AM J OBSTET GyNeCOL 1981;78:111-3.

8. Haas AJ. Palatal expansion: just the beginning of dentofacial orthopedics. AM J ORTHOD 1970;57:219-55.

9. Tversky A, Kahneman D. Judgment under uncertainty: heuristics and biases. Science 1974;185:1124-31.

10. Weintraub JA, Burt BA. Oral health status in the United States: tooth loss and edentulism. J Dent Educ 1985;49:368-76.

11. National Caries Program, National Institute of Dental Research. The prevalence of dental caries in the United States children 1979-1980. National Dental Caries Prevalence Suryey. DHHS Publication No. (NIH)82-2245, 1981.

12. National Caries Program, National Institute of Dental Research. Dental treatment needs of United States children 1979-1980. National Dental Caries Prevalence Survey. DHHS Publication No. (NIH)83-2246, 1982.

Reprint requests to:

Dr. Peter S. Vig

Department of Orthodontics and Pediatric Dentistry

Center for Human Growth and Development

University of Michigan

Ann Arbor, MI 48109 